\title{
Impact of high-speed diesel drop trains: Pursuing cleaner diesel engines
}

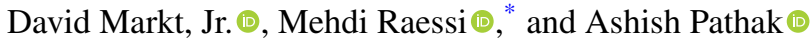 \\ Department of Mechanical Engineering, University of Massachusetts Dartmouth, \\ Dartmouth, Massachusetts 02747, USA \\ Seong-Young Lee \\ Department of Mechanical Engineering-Engineering Mechanics, \\ Michigan Technological University, Houghton, Michigan 49931, USA \\ Roberto Torelli $\odot$ \\ Argonne National Laboratory, Lemont, Illinois 60439, USA \\ (Received 9 August 2021; published 15 November 2021)
}

\begin{abstract}
This paper is associated with a video winner of a 2020 American Physical Society's Division of Fluid Dynamics (DFD) Milton van Dyke Award for work presented at the DFD Gallery of Fluid Motion. The original video is available online at the Gallery of Fluid Motion, https://doi.org/10.1103/APS.DFD.2020.GFM.V0052.
\end{abstract}

DOI: 10.1103/PhysRevFluids.6.110508

The splashing of liquid drops has been widely studied as this phenomenon is prevalent in many modern industries. In internal combustion engines (ICEs), specifically diesel engines, liquid fuel is sprayed at high pressure into the combustion chamber forming a dense cloud of fuel drops. The diesel drops impact the surface at sizes nearing a single micron with impact velocities upward of $100 \mathrm{~m} / \mathrm{s}$ [1], making this problem extremely difficult to study experimentally. Consequently, drop impingement under ICE conditions is still largely misunderstood [2]. This knowledge gap has large ramifications in the automotive industry since the splashing of fuel drops, and subsequent air-fuel mixing, has a direct effect on combustion efficiency and pollutant emission [3].

In this study, we leverage the capabilities of an in-house message passing interface parallel multiphase flow solver [4] to investigate the impingement of a monodispersed diesel drop train onto a preexisting diesel film of $0.31 \mu \mathrm{m}$ thickness. We used ICE relevant impact conditions that include drop diameter $D=5.97 \mu \mathrm{m}$ and impingement velocity $V_{0}=77 \mathrm{~m} / \mathrm{s}$, taken from [1]. The drop train is depicted in Fig. 1 with a drop spacing of $S=4.16 D$ which corresponds to an impact frequency of $3.103 \mathrm{MHz}$, and leads to splashing based on the work of Yarin and Weiss [5]. Under engine relevant conditions, the density, surface tension, and kinematic viscosity of diesel are $\rho=848 \mathrm{~kg} / \mathrm{m}^{3}$, $\sigma=20.8 \times 10^{-3} \mathrm{~N} / \mathrm{m}$, and $v=6.25 \times 10^{-7} \mathrm{~m}^{2} / \mathrm{s}$, respectively. The impact conditions correspond to Reynolds $(\operatorname{Re}=V D / \nu)$ and Weber $\left(\mathrm{We}=\rho V^{2} D / \sigma\right)$ numbers of $\operatorname{Re}=736$ and $\mathrm{We}=1443$. The computational domain dimensions are $4.19 D \times 4.19 D \times 3.22 D$, sufficiently large to capture the splashing phenomena. In the simulation a spatial resolution of 160 cells per $D$, or a uniform mesh resolution of $\Delta x=0.0375 \mu \mathrm{m}$, was used.

\footnotetext{
*mraessi@umassd.edu
}

Published by the American Physical Society under the terms of the Creative Commons Attribution 4.0 International license. Further distribution of this work must maintain attribution to the author(s) and the published article's title, journal citation, and DOI. 


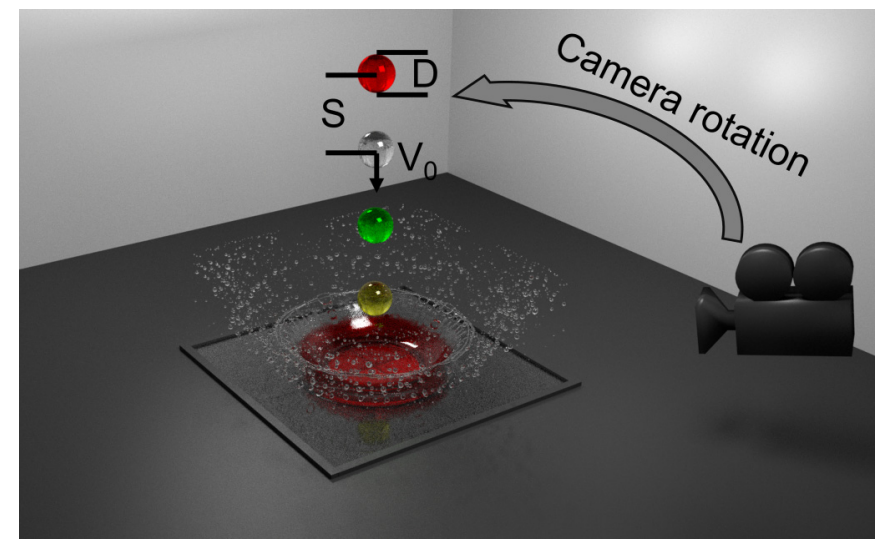

FIG. 1. Simulation of a diesel drop train impacting a liquid film showing important parameters (drop diameter $D$, drop spacing $S$, and impact velocity $V_{0}$ ) and the camera rotation used to generate the images in Fig. 2.

In Fig. 1, three drops have impacted the liquid film and four drops are seen approaching the impingement location. To facilitate analysis of the splashing process, each drop is tagged with a distinct passive scalar color function [6], allowing each drop to be tracked in a sharp fashion. A detailed description of the passive scalar routine is presented in [6]. As seen in Fig. 1, four repeating passive scalars are used to track individual drops, colorless, red, yellow and green, respectively. The preexisting film and the first two drops are colorless.

For the sake of brevity, this article only highlights the stages of liquid breakup and secondary droplet formation, shown in Fig. 2, for the above diesel drop train simulation. Our analyses on the secondary droplet distributions and splashed mass ratio are included in the Gallery of Fluid Motion video https://doi.org/10.1103/APS.DFD.2020.GFM.V0052 and in [7]. The secondary droplet characterization was done using the algorithm presented in [8].

The images in Fig. 2 were obtained using a rotating camera angle as depicted in Fig. 1 and show the impingement of the first seven drops within the drop train. Three distinct phases of secondary droplet formation are observed in a repeating process: First, a ring of small secondary droplets is ejected from the lamella rim shortly after impingement. This produces some of the smallest secondary droplets as seen in Fig. 2(a). As the lamella continues to stretch, instabilities in the upper lamella produce a ligament structure, appearing to occur at a specific wavelength as seen in Fig. 2(c) for the impingement of the third drop (red). The ligaments at the top of the lamella then form cusps from which jets of secondary droplets are ejected, marking the second phase of secondary droplet formation. Here larger secondary drops are produced as seen for the impingement of the fourth drop in Fig. 2(d). Finally, in Fig. 2(b) the detachment of the lamella is shown, forming a lower lamella rim. As the lower rim travels away from the impingement location, it is stretched until fracturing into large secondary droplets as depicted in Fig. 2(e) for the impingement of the fifth drop (green). This is the final phase of secondary droplet formation and produces the largest secondary droplets. Figure 2(f) clearly shows that the cloud of secondary droplets is becoming increasingly more dense. Furthermore, the use of passive scalars shows that the lamella rim is composed of liquid from the previously impinged drop, while the lower lamella is composed of liquid from the impinging drop itself as seen in Figs. 2(c) and 2(d).

Secondary droplet characteristics have a direct impact on fuel combustion. Uncovering the mechanisms behind secondary droplet formation is necessary to ultimately develop predictive models for secondary droplet size distributions that can be reliably used in combustion simulations for design of cleaner diesel engines. 

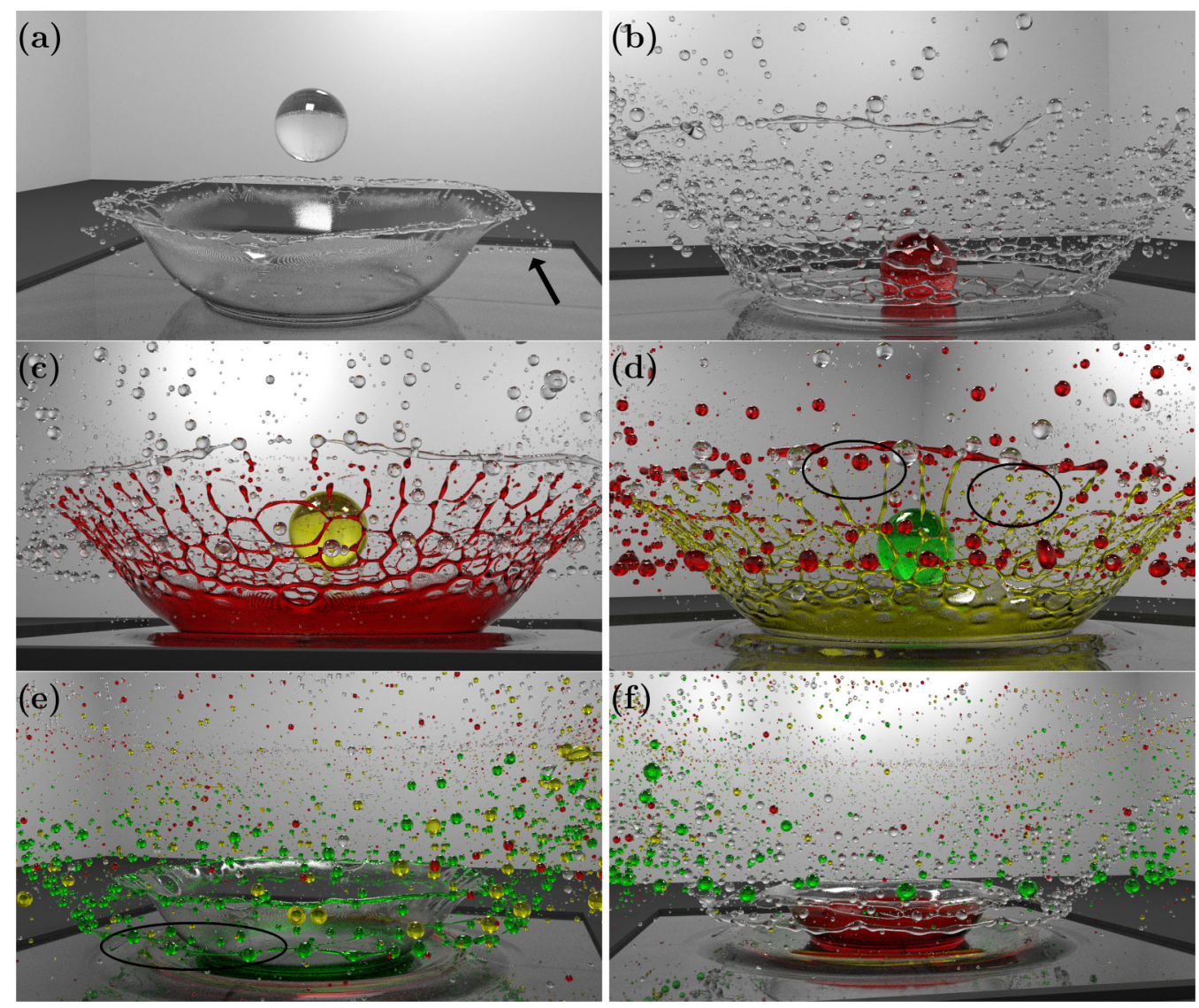

FIG. 2. High-resolution ( $\Delta x=0.0375 \mu \mathrm{m}$ ) simulation results of a diesel drop train impacting a preexisting liquid film. Four passive scalars are used to track each impinging drop with a repeating color scheme of clear, red, yellow, and green. Three distinct phases of secondary droplet formation are identified in the images.

This material is based upon work supported by the Department of Energy, Office of Energy Efficiency and Renewable Energy and the Department of Defense, Tank and Automotive Research, Development, and Engineering Center, under Award No. DE-EE007292, and the Massachusetts Clean Energy Center. The computations were performed on resources provided by NSF XSEDE through Grants No. ENG170004 and No. ENG170008.

[1] D. Markt, Jr., R. Torelli, A. Pathak, M. Raessi, S. Som, R. Scarcelli, S.-Y. Lee, and J. Naber, Using a DNS framework to test a splashed mass sub-model for Lagrangian spray simulations, SAE Report No. 2018-01-0297 (2018), https://doi.org/10.4271/2018-01-0297.

[2] G. Liang and I. Mudawar, Review of mass and momentum interactions during drop impact on a liquid film, Int. J. Heat Mass Transf. 101, 577 (2016).

[3] M. Drake, T. Fansler, A. Solomon, and G. Szekely, Piston fuel films as a source of smoke and hydrocarbon emissions from a wall-controlled spark-ignited direct-injection engine, SAE Report No. 2003-01-0547 (2003), https://doi.org/10.4271/2003-01-0547.

[4] A. Pathak and M. Raessi, A 3D, fully Eulerian, VOF-based solver to study the interaction between two fluids and moving rigid bodies using the fictitious domain method, J. Comput. Phys. 311, 87 (2016). 
[5] A. Yarin and D. Weiss, Impact of drops on solid surfaces: Self-similar capillary waves, and splashing as a new type of kinematic discontinuity, J. Fluid Mech. 283, 141 (1995).

[6] D. Markt, Jr., A. Pathak, and M. Raessi, Advanced computational simulations of surface impingement of a train of ethanol drops: Pathway to developing spray-wall interaction submodels, Comput. Sci. Eng. 20, 56 (2018).

[7] D. Markt, Jr., M. Raessi, S.-Y. Lee, and X. Zhu, High-speed impact of micron-sized diesel drop trainsSplashing dynamics, secondary droplet formation and effects of pre-existing film thickness, Phys. Fluids 33, 102120 (2021).

[8] D. Markt, Jr., A. Pathak, M. Raessi, S.-Y. Lee, and R. Torelli, Computational characterization of the secondary droplets formed during the impingement of a train of ethanol drops, Int. J. Engine Res. 21, 248 (2020). 